Biol Philos

DOI 10.1007/s10539-012-9334-2

\title{
Cancer cells and adaptive explanations
}

Pierre-Luc Germain ( pierre.germain@ieo.eu )

European School of Molecular Medicine (SEMM), European Institute of Oncology (IEO), Università degli Studi di Milano

Via Adamello 16, 20139 Milan, Italy

+3902574303.200

\begin{abstract}
:
The aim of this paper is to assess the relevance of somatic evolution by natural selection to our understanding of cancer development. I do so in two steps. In the first part of the paper, I ask to what extent cancer cells meet the formal requirements for evolution by natural selection, relying on Godfrey-Smith's (2009) framework of Darwinian populations. I argue that although they meet the minimal requirements for natural selection, cancer cells are not paradigmatic Darwinian populations.

In the second part of the paper, I examine the most important examples of adaptation in cancer cells. I argue that they are not significant accumulations of evolutionary changes, and that as a consequence natural selection plays a lesser role in their explanation. Their explanation, I argue, is best sought in the previously existing wiring of the healthy cells.
\end{abstract}

Keywords: cancer, adaptation, natural selection, explanation, Darwinian populations. 


\section{Cancer cells and adaptive explanations}

It is very common to read that cancer is "a disease of clonal evolution within the body" (Merlo et al. 2006, p. 924). This idea has gained most of its popularity in the last thirty years or so, following the publication of well-developed evolutionary accounts of cancer development (most famously Cairns 1975 and Nowell 1976; for a review of the landmark publications, see Attolini and Michor 2009). According to some contemporary authors, "the fundamental problems of neoplastic progression and cancer therapy are also problems of evolutionary biology" (Merlo et al. 2006, p. 933). They are not simply saying that the study of the somatic evolution of cancer cells is a research avenue worth exploring - a claim that most scientists would certainly second. Their claim is much stronger:

"consensus emerged that somatic (within-body) cellular selection and evolution is the fundamental process by which neoplasms arise, acquire malignancy, and evade therapeutic interventions"

(Pepper et al. 2009, p. 62, emphasis added)

Despite this apparent consensus, the authors note, this view "has seldom been integrated into biomedical research" (Pepper et al. 2009, p. 63). Studying cancer as an evolutionary phenomenon, they claim, would improve both our understanding of its development and clinical outcomes (Gatenby and Gillies 2008; Gerlinger and Swanton 2010; Pepper et al. 2009). Some go further and claim that it is necessary in order "to transform cancer research into a rational and predictive science" (Attolini and Michor 2009, p. 24).

This opinion has not fallen on deaf ears. Despite thirty years of intense research through Cancer Chemotherapy National Service Center, the American medical community concluded in the 1980's that "we are losing the war on cancer" (Bailar and Smith 1986, p. 1226). Since the 1990's, after even more massive investments in cancer research and prevention programs, one can at least claim that clinical outcomes are getting better, but progress is painfully slow, and cancer continues to be a major killer (Eheman et al. 2012). In this context, the promises of an evolutionary perspective on cancer - which purports to explain the very urgent problem of tumour relapse - are attractive. The new approach, if heeded, would imply a major reorientation of research programmes in molecular oncology, and a reshaping of clinical practice (Gatenby and Gillies 2008; Gillies et al. 2012; Heng et al. 2010). It is therefore important to evaluate the reasons we have to support these claims. The aim of this paper is to assess, from a philosophical point of view, the potential relevance of somatic Darwinian processes for the understanding of cancer development ${ }^{1}$.

To ask whether cancer development should be seen as an evolutionary phenomenon can mean two different things: 1) whether (and to what extent) Darwinian processes occur among cancer cells; and 2) whether (and to what extent) these processes, if they occur, are useful for the understanding of cancer cells and their features. I address both questions respectively in the two main parts of the paper.

In the first part, I ask whether cancer cells meet the formal requirements of evolution by natural selection, applying Godfrey-Smith's (2009) recent account of Darwinian populations. I show that with respect to this framework, cancer cells are much closer to normal somatic cells than is generally suggested, and argue that although they fulfill the minimal requirements for natural selection, they are

1 By speaking of cancer development, I explicitly wish to avoid discussions of cancer in light of the evolution of multicellular life (Frank 2007; Frank et al. 2003; Hutchinson et al. 2003; Buss 1987). What I am interested in is the evolution of cancer cells within a given tumour. This also means that I exclude the very special cases of transmittable cancers (see for instance Belov 2012). 
not paradigmatic Darwinian populations.

This conclusion suggests that cancer cells are unlikely to develop complex adaptations - that is, adaptations that are an accumulation of evolutionary changes. In the second part of the paper, I therefore examine the best candidate adaptations in cancer cells to determine whether they are the result of cumulative evolution. I argue that, for the most part, they are not. Furthermore, I discuss the explanatory relevance of natural selection for these cases. While I do not deny the role of natural selection, I propose that alternative explanations based on the original molecular architecture of the cell do much more explanatory work than invoking natural selection.

\section{Are cancer cells Darwinian populations?}

The best empirical evidence for natural selection within a tumour probably comes from phylogenetic studies of cancer (sometimes called "tumour archaeology"), which attempt to reconstruct a schematic history of the appearance and spread of genetic alterations (see for example Nik-Zainal et al. 2012; Anderson et al. 2011; Sabatino et al. 2008). While natural selection is not the only possible cause of patterns of genetic heterogeneity (see Nik-Zainal et al. 2012a on DNA damage and repair processes), it is quite uncontroversial by now that cancer cells undergo differential persistence. Tumours consist of different subclones, and some lineages go extinct while others thrive. The real issue is not whether natural selection occurs within a tumour, but what we can expect out of it. In itself, some differential persistence is insufficient for paradigmatic evolution (it could be entirely accidental), and assessing the relevance of Darwinian processes in cancer development requires an inquiry into the conditions of possibility of evolution by natural selection.

\section{Godfrey-Smith's framework}

I claim that the conception of cancer development as an evolutionary phenomenon is often overrated in the scientific literature, and there are two major reasons for this. The first is an unfortunate conflation of natural selection and evolution by natural selection, with a jump from evidence of the former to inference of the latter. The second reason is a simplistic understanding of the formal conditions for evolution by natural selection, limited to a rather weak reading of the traditional requirement of inherited variations in fitness (for instance Ridley 2007). Indeed it is common to read in papers about cancer that " $[\mathrm{t}]$ he heritable variation of reproductive success in a population is necessary and sufficient to cause natural selection" (Merlo et al. 2006, p. 927, emphasis added; see also Pepper et al. 2009; Heng et al. 2010). Although these classical criteria are highly relevant to evolution by natural selection, many authors (including Godfrey-Smith 2009) have noted that there are counter-arguments to both the necessity and the sufficiency of these "ingredients". The cells in our body, to start with, meet these requirements at least to some degree, but most of them do not undergo paradigmatic evolution by natural selection (Godfrey-Smith 2009, p. 56). The conditions of possibility of evolution by natural selection are more complex; first because they depend on more than the classical conditions, and second because each condition can be met to various degrees. Variation, for instance, is required for evolution by natural selection, but a population is not said either to have or not to have variation - it has a certain amount of variation. Evolution is impossible without a minimum of variation, but too much of it will lead to a scenario of "error catastrophe" in which no evolution is possible. 


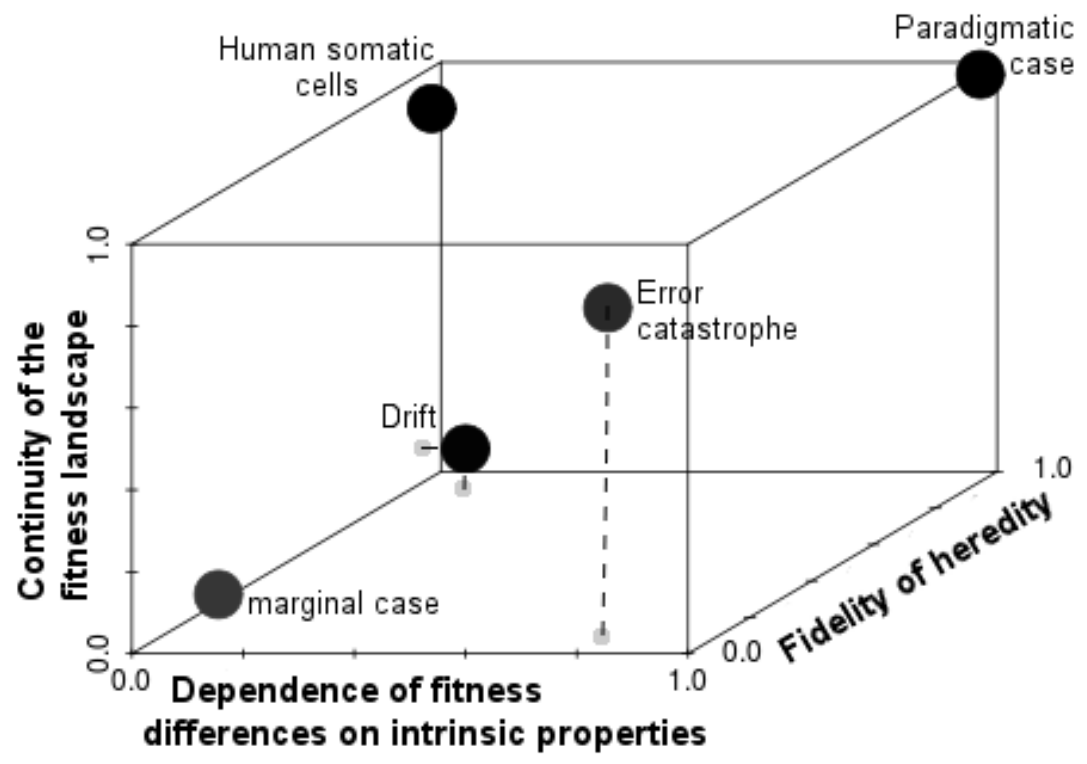

Godfrey-Smith (2009) pays close attention to these complexities. He develops the concept of a Darwinian population, which is a population of entities that can be expected to undergo evolution by natural selection. He then proposes to view the factors that are relevant to whether a population is Darwinian as different dimensions of a space. Figure 1 shows a representation of this space (taking into account only three dimensions), with sample kinds of populations. For our purposes, the most important dimensions are the following:

$\begin{array}{lll}\mathbf{H} & - & \text { Fidelity of heredity } \\ \mathbf{V} & - & \text { Abundance of variation } \\ \mathbf{C} & - & \text { Continuity, or smoothness of the fitness landscape } \\ \mathbf{S} & - & \text { Dependence of reproductive differences on intrinsic character } \\ \mathbf{G} & - & \text { The degree of reproductive specialization } \\ \mathbf{I} & - & \text { Integration, or the extent of mutual dependence }\end{array}$

I will describe each dimension in more detail when applying them to the case of cancer. For the moment, what is important to note is that each dimension (for instance the classical criterion of variation) varies along a continuum, and likewise the extent to which a population is Darwinian varies according to its position in this space. Some populations are more Darwinian than others. Although there is no clear-cut threshold, Godfrey-Smith identifies different subsets of populations, with all shades of grey in-between. Some collections of entities cannot possible undergo natural selection, and those are not Darwinian populations. Minimal Darwinian populations satisfy the traditional requirements mentioned above, at least minimally. While they can undergo natural selection, some of these populations are Darwinian in a "trivial" way, and therefore

"the process described in the minimal concept should not be installed as "the" Darwinian process. Significant Darwinian processes have extra features, and these can in some cases be described abstractly. So within the area staked out by the minimal concept, we can identify a category of paradigm Darwinian populations. This is the kind of system that can produce novel and complex organisms, highly adapted to their circumstances." (Godfrey-Smith 2009, p. 6, emphasis added)

I will return to the issue of complex adaptations in the second part of this paper. For the moment, what matters is that assessing the significance of natural selection for the study of cancer requires more than asking whether natural selection obtains in tumours, or whether cancer cells are a Darwinian population. The question is rather what kind of Darwinian population cancer cells are, or at least how 
Darwinian they are $^{2}$.

Godfrey-Smith's framework is therefore more precise in its understanding and assessment of the conditions of possibility of evolution by natural selection. Furthermore, it is particularly well suited to address evolutionary issues between different levels of biological organization. When dealing with populations at multiple levels, for instance the population of cells in my body and the human population, the extent to which the population at each level is Darwinian depends on how Darwinian those at other levels are. According to Godfrey-Smith, multicellular organisms could be entities of a Darwinian population only by de-Darwinizing their component cells at least to some extent. Darwinian processes at the cellular level impedes Darwinian processes at the level of organisms. Two of GodfreySmith's dimensions, integration $(I)$ and reproductive specialization $(G)$, are precisely meant to capture this inter-dependency and clarify the ways in which "[o]ne Darwinian population can 'de-Darwinize' others." (Godfrey-Smith 2009, p. 9). As these dimensions will be of particular relevance to the case of cancer, it is worth sketching them here.

The degree of integration $(I)$ is "a summary of such features as the extent of division of labor, the mutual dependence (loss of autonomy) of parts, and the maintenance of a boundary between a collective and what is outside it" (Godfrey-Smith 2009, p. 93). Our body has a very high level of integration, for most cells depend on those of the digestive tract to digest nutrients, or on those of the immune system to protect them from pathogens, etc. This interdependency prevents effective competition between somatic cells, but in doing so it allows the whole organisms to undergo Darwinian processes. Likewise, reproductive specialization $(G)$ makes somatic cells depend on germ cells for the long-term reproduction of their genes, impairing Darwinian processes at the level of cells and enabling it at the level of organisms. As a consequence somatic cells are "evolutionary dead-ends", and the same applies within the lifetime of the organism: in general tissue architecture displays the same reproductive specialization. In most tissues, differentiated cells have a very short lifespan and limited replication potential, and regeneration is assured by a small population of stem cells ${ }^{3}$. Within the timeframe of the organism's lifespan, terminally differentiated cells are also evolutionary dead-ends, while stem cells can persist throughout our lifetime ${ }^{4}$.

In discussing these dimensions, Godfrey-Smith mentions cancer explicitly. According to him, multicellular organisms evolved by de-Darwinizing their somatic cells, and cancer is a reversal of this process. Cancer is a "consequence of cell-level Darwinian processes" (Godfrey-Smith 2009, p. 101). Although Godfrey-Smith clearly considers cancer to be essentially an evolutionary phenomenon, he does not address the issue in any detail. Here, I attempt to do so, and closer inspection reveals a more complex story.

The assessment of how Darwinian a population is implies measuring the position of cancer cells on each dimension. As it would be extremely complex, if at all possible, to do so in a quantitative or absolute manner, I suggest to think of their position relative to normal somatic cells. Godfrey-Smith's interpretation implies that the different constraints placed on somatic cells to avoid Darwinian processes are somehow lifted in cancer cells. My strategy, therefore, is to inspect those differences, and

2 Rather than a simple gradient of more or less Darwinian populations, the multi-dimensionality suggests that there are different kinds of Darwinian processes. Godfrey-Smith (2009) does not explore this in depth, and neither will I do so here.

3 These stem cells divide asymmetrically to produce an identical daughter (so that the stem cell pool persists) and a progenitor cell (which proliferates very quickly but only up to a certain point). Grompe (2012) provides a short but enlightening review of tissue stem cells and their technical implications.

4 In fact, many authors have suggested that tissue architecture is an adaptation against cancer (Frank and Nowak 2004; Frank et al. 2003). 
investigate whether the changes occurring in carcinogenesis correspond to significant shifts in the dimensions of his framework. Therefore, among the different dimensions characterized by GodfreySmith, I concentrate on those that are especially expected to be different in cancer cells. After a brief primer on cancer, I will address each of the relevant dimensions.

\section{Cancer cells and models of carcinogenesis}

Cancer is uncontrolled cell growth, but the different types of cancer are extremely heterogeneous. Despite this heterogeneity, cancer is characterized by features at the cellular level (mostly), termed the "hallmarks of cancer" (Hanahan and Weinberg 2011; Hanahan and Weinberg 2000). These features can be summarized in the following way:

- $\quad$ self-sufficiency in growth signals and insensitivity to inhibition signals

- evasion of apoptosis (cancer cells do not die or go into senescence when they should)

- immortality (cancer cells replicate indefinitely, avoiding Hayflick's limit)

- angiogenesis (cancer cells induce the creation of blood vessels providing nutrients and oxygen)

- invasion (cancer cells can grow independently from tissue anchoring)

According to the accepted model of carcinogenesis - the multiple-hit theory (Armitage and Doll 1957; Vogelstein and Kinzler 1993) - cells must acquire a series of (semi-)specific mutations to become cancerous. The hallmarks of cancer, therefore, were presented as an abstract representation of the kinds of mutations required for cancer (Hanahan and Weinberg 2000). The reasoning rests on the assumption that those features are not present in normal somatic cells. While this is true of most cells, normal stem cells have a lot in common with cancer cells, and this similarity is the ground for an alternative model of cancer development: the Cancer Stem Cell (CSC) model.

The CSC hypothesis is a model of cancer development originally developed for leukaemia (for reviews of the model, see Frank et al. 2010; Visvader and Lindeman 2012). The model initially proposed that cancer cells are abnormally stopped in their differentiation process. Nowadays, it is taken to mean that a very small subpopulation of tumour cells (the CSCs), endowed with stem cell-like properties, accounts for the maintenance and progression of the tumour. The model is therefore independent of whether CSCs have these stem cell-like properties because of serial mutations which reverted their differentiation, or also because the oncogenic transformation occurred in a stem cell which already had some of these features. However, the latter hypothesis - called the cell-of-origin hypothesis - is often joined to the CSC hypothesis (see Barker et al. 2009 for evidence of the stem-cell origin of intestinal cancer). In any case, only CSCs have the potential for indefinite self-renewal, but they are relatively quiescent (they do not replicate often), dividing mostly asymmetrically, like normal stem cells, to give rise to short-lived but more proliferative cells. Without the CSCs, proliferation would quickly stop, and this idea has nourished the hope of finding a "magic bullet" - a targeted drug.

Today it is widely accepted that at least myeloid leukaemia follows a CSC model, and CSCs have also been reported in breast cancer (Pece et al. 2010; Dick 2003), brain tumours (Singh et al. 2003), and many other types of cancer (Frank et al. 2010). Even in these tissues, however, the issue is still debated. The great hype surrounding the model, which is evident in the scientific literature, has promoted the idea that all cancers follow a CSC model. In fact, however, it is still far from an established fact in most solid cancers (see Maenhaut et al. 2010). Cancers like melanoma, that display huge genetic instabilities and develop from tissues without a very entrenched developmental hierarchy, 
are very unlikely to follow a CSC model in any meaningful way. The last emphasis is important, because just like the question of whether cancer is an evolutionary phenomenon, whether a cancer follows a CSC model is a matter of degrees. This is a direct consequence of the vagueness of the CSC model, which specifies neither how rare nor how stable the cancer stem cells should be. Both models of carcinogenesis are just that - models or approximations. As a commentator noted, "the collective evidence suggests that various tumours may span the spectrum between the extremes represented by the two models" (Adams and Strasser 2008, p. 4018). This is often overlooked by the literature, but it is a very important point both for the meaning of the CSC model and, as I will show in a moment, for the prospect of evolution.

The upshot of this short overview is that different types of cancers will most likely occupy slightly different positions in Godfrey-Smith's framework. It would be impracticable to address each cancer type here, but when there will be significant differences I will consider the two extreme cases: cancers that are paradigmatic of the CSC model (like myeloid leukaemia) versus those that are not (like, most probably, melanoma).

With this background laid out, I will now attempt to locate cancer cells in Godfrey-Smith's spatial framework by addressing, in turn, the most relevant to the case of cancer.

\section{Abundance of variation $(V)$ and fidelity of heredity $(H)$}

With some exceptions ${ }^{5}$, mammalian somatic cells are very stable genetically. Cancer cells share the mechanisms of heredity of their normal counterparts, but they are known for their genetic instability: mutations are much more frequent, and sometimes reach surprising levels (see Stephens et al. 2011 for a dramatic example; for a review, see Stratton 2011). Cancer cells often exhibit thousands of genetic modifications, including major genetic aberrations such as chromosomal loss, gain or fusion. Variation $(V)$ is considerably more abundant than in normal somatic cells, and certainly sufficient for evolutionary change. In fact the extent of variation might suggest that fidelity of heredity $(H)$ is dangerously low, but there is compelling evidence against this view. In most examples, the least genetically stable cell populations are also the most aggressive (see for instance Anderson et al. 2011; Ye et al. 2009; Maley et al. 2006), which strongly argues against an "error catastrophe" scenario.

The correlation between heterogeneity of the tumour population and bad prognosis has long been known to the medical community. While it is difficult to say whether the bad prognosis and the heterogeneity are both consequences of a common cause, namely the tumour being in a more advanced state, there seems to be an independent association (Maley et al. 2006). This is often explained from an evolutionary point of view: lower variation would mean fewer possibilities for evolutionary change, and consequently for the development of resistance to selection pressures such as therapy ${ }^{6}$. It must be noted, however, that the correlation is not in itself evidence for the activity of natural selection. In fact,

5 B-cells are systematically cited as the exception to this rule, but it must be noted that the list of "exceptions" is beginning to grow at a disturbing pace. See for instance the work of Gage's lab on LINE-1 retrotransposons, most importantly in the central nervous system (Singer et al. 2010).

6 Proponents of the CSC model argue that heterogeneity and bad prognosis are both consequences of a common cause: the cancer stem cells being blocked early in the differentiation tree. The less differentiated they are, the more their progeny can differentiate heterogeneously, and the greater their capacity for self-renewal (hence the severity of the cancer). However, the two explanations are not aimed at the same kind of heterogeneity. The evolutionary explanation explains the relevance of genetic heterogeneity, while the CSC model is aimed at phenotypic heterogeneity - an heterogeneity that is the consequence of varied differentiation rather than mutation. 
past selection implies a reduction of heterogeneity ${ }^{7}$, so that very fit (hence aggressive) populations of cancer cells should be less heterogeneous, contrary to the observed correlation. What the correlation does suggest, is that therapy will be a strong selection pressure, and that genetically heterogeneous tumours have better chances of getting through it. In any case, different strands of evidence suggest that the instability of cancer cells helps rather than hampers their evolution (see also Beckman and Loeb 2006). Although it is still unclear whether fidelity of heredity is ideal for evolution, we can safely consider it within the required range. The next dimensions, however, are not so easy to settle.

\section{Continuity or smoothness of the fitness landscape $(C)$}

Godfrey-Smith presents this dimension through the metaphor of the fitness landscape:

"We imagine properties of organisms (or sometimes, populations) represented in several dimensions, and fitness represented with another dimension, visualized as height. So mountains (if there are any) correspond to areas of high fitness, valleys to areas of low fitness. [...] we have a "smooth" landscape when similar organismic properties are associated with similar fitness values." (Godfrey-Smith 2009, p. 57)

The fitness landscape is discontinuous or rugged if small changes in a trait lead to dramatic consequences on fitness. The magnitude of the effect is not very relevant in itself: what matters is that the slope between two points can be more or less accurately predicted from the slope between nearby points. The logic is that an evolutionary process with good fidelity of heredity and adequate variation will tend to climb up nearby hills of the fitness landscape, provided that it is smooth enough. In other words, smooth hills are evolutionary attractors: nearby states are attracted to it, so that they are likely to be found, and random departures from it will tend to return to it. In addition, smoothness allows to leave local hills and explore other regions of the landscape without immediately falling into a "hole" (i.e. without very important losses of fitness). When the landscape is rugged, however, there is little margin for exploration and the number of evolutionary roads to a peak is reduced. According to Godfrey-Smith, while high continuity is not strictly required for minimal natural selection, it is needed for paradigmatic evolutionary change. ${ }^{8}$

Given the abstract and metaphorical nature of the fitness landscape, as well as the difficulty of clearly individuating the dimensions, $C$ is particularly difficult to assess. Nevertheless, there are reasons to believe that if it is any different between cancer cells and normal cells, the fitness landscape should be more rugged in cancer cells. This is best seen by looking at the robustness of cellular states. As Godfrey-Smith (2009) explains, our cells have evolved many mechanisms, such as heat-shock proteins, which make development more robust to both internal and environmental variations, thereby increasing $C$. The robustness of differentiation into specific cell types (in other words, the smoothness of the epigenetic landscape) likewise increases $C$, for it allows some parameters to vary slightly without altering development too much. Systems biology has proposed to see cell types as attractor states, so that small variations around a state tend to converge to it. However, Huang (2011) proposes that the cancerous state of a cell, as opposed to normal cell types, is a "non-evolved attractor": akin to spandrels (Gould and Lewontin 1979), cancerous states are structural consequences of the epigenetic landscape. As they are located in a portion of the epigenetic landscape not normally used, they have been "out of

7 For a discussion of this issue, see Shibata's (2006) comment on the results presented by Maley et al. (2006)

8 It should be immediately obvious that different traits or regions of the fitness landscape will be continuous to different degrees. There will be areas that are more "rugged" than others. Different features of a population might fare differently with respect to dimension $C$, and hence might be expected to evolve at different rates and to different extents. In principle, this does not prevent us from assessing $C$ for the whole landscape, although in practice this is beyond reach. What it does mean, however, is that this dimension could be of use in a more fine-grained analysis. 
reach for fine-tuning by organismal evolution" (Huang 2011, p. 194). In other words, while metazoan evolution has in general worked to make the normal regions of the epigenetic landscape smoother, thereby increasing robustness and also making the local fitness landscape smoother, no such "polishing" has occurred for cancer states. This suggests that if there is a difference in $C$ between healthy and cancerous cells, we should expect a lower $C$ in the latter.

\section{Integration (I) and Reproductive Specialization $(G)$}

Cancer cells are generally depicted as "renegade cells", or "selfish cells", in comparison to our normal somatic cells which live in a highly integrated community. While there is certainly some truth in this description (indeed, cancer cells often bring about the death of their host), it must be tempered: in reality, cancer cells form "complex tissues" (Hanahan and Weinberg 2000, p. 60), or even "organlike populations" (Miller et al. 2005, p. 25; see also Soto and Sonnenschein 2004; Bissell and Radisky 2001), showing a surprising degree of integration'. In Godfrey-Smith's framework, the classical example of integration $(I)$ is division of labour, which arguably does not obtain in tumour cells. Nevertheless, it has been demonstrated that in epithelial cancers (the majority of cancers), progression often depends on the interaction between cancer cells and normal epithelial cells (Bhowmick and Neilson 2004; Bissell and Radisky 2001). Even cancer cells exhibit some degree of mutual dependency. For example, one of the hallmarks of cancer is angiogenesis, the process by which cancer cells induce the formation of new blood vessels, thereby ensuring the tumour a flow of nutrients and decreasing its concentration of carbon dioxide. In the past ten years, this process has received a lot of attention as a potential target for adjuvant therapy (see for instance Corada et al. 2002). For our discussion, what matters is that the formation of new blood vessels requires the orchestrated action of many cells, and affects the tumour as a whole: blood vessels do not serve single cells, but rather a whole region of the tumour. Therefore, it seems unlikely that cells promoting angiogenesis can gain a significant fitness advantage over the nearby cells - those with which they directly compete. To put it in Godfrey-Smith's terms, the cells in a tumour might be too integrated to fully compete with one another. Obviously, this does not apply to all fitness differences, but it might be extended to some other hallmarks, and it is especially relevant for the interactions between the tumour and the immune system. In general, the growing recognition that tumours display a higher level of integration than was originally thought makes our picture of cancer cells as renegade cells clearly over-simplified.

The most important kind of mutual-dependency is arguably reproductive specialization $(G)$. Within the confines of our body, this takes the form of tissue architecture. One might think that this is irrelevant to cancer cells, generally pictured as outlaws of tissue architecture, proliferating for their own good. However, the Cancer Stem Cells (CSC) model presented earlier suggests that many cancers have, in fact, a tissue architecture. And this is most crucial to the somatic evolution of cancer cells.

Even in a cancer which follows the CSC model perfectly, some differentiated cells (non-CSC) will have more reproductive success than others, and therefore we may expect natural selection to occur within these populations. However, since they have a very limited replication potential, cumulative evolution is very unlikely. Even if it occurred, its product would quickly be lost and would certainly be of no clinical relevance. Though in a very short time frame every cancer cell reproduces, on a slightly larger scale (i.e. a dozen replications), more relevant to oncology, only CSC can undergo long-term reproduction. This corresponds to a high reproductive specialization, but it does not exclude significant Darwinian processes among the CSC population. These, however, are by definition very few, and as quiescence is among their properties, they seldom reproduce. Moreover, the model predicts

9 For a philosophical discussion of this issue, see Bertolaso 2011. 
that when they do reproduce, it is to give rise to, on the one hand, an identical daughter, and on the other a differentiated lineage. This means that the CSC population does not expand, and that the only expanding lineage is reproductively limited. In both subpopulations, therefore, cumulative evolution seems very unlikely.

However, as I mentioned already the CSC model is an idealization, and fits actual cancers only to some degree. It does not specify, for instance, how rare the CSCs must be. If there are only a couple of CSCs in their respective niche, evolution seems impossible. But what if $10 \%$ of the tumour cells are cancer stem cells? And what if, as evidence seems to suggest, they do not only divide asymmetrically, but also symmetrically, increasing the CSC pool rather than giving rise to a differentiated lineage? Even if the other cells did not evolve, these $10 \%$ might. Likewise, what if (as I suggest in the next section) cells can switch from and to a CSC-state? The probability of such events will vary a lot across contexts, and so will the degree of reproductive specialization. In myeloid leukaemia, $G$ is very high; it is already slightly lower in some other forms of leukaemia, and it is certainly very low in melanoma. For most cancers, it is reasonable to consider the degree of reproductive specialization sufficiently low for minimal Darwinian processes, but not low enough to rank cancer cells as paradigmatic Darwinian populations.

\section{Dependence of fitness differences on intrinsic characters $(S)$}

Reproductive specialization and mutual-dependency between cells are special ways of making the fate of a cell more dependent on factors external to it, but they are not the only one. Godfrey-Smith characterizes this more general kind of dependency in dimension $S$ :

" $S$ is the extent to which 'realized' fitness differences in a population are tied to differences in intrinsic character. [...] If extrinsic features are most of what matters to realized fitness - if intrinsic character is not very important — then other than this physical wandering, not much can happen.” (Godfrey-Smith 2009, pp. 54-55 )

Of course, virtually every fitness difference depends on the environment, and therefore is not entirely intrinsic. As the author emphasizes, however, some differences are almost completely extrinsic:

"When differences in reproductive output depend mainly on things like location - on who is in the right place at the right time - and these extrinsic differences are not the result of other intrinsic features - we have a low S." (Godfrey-Smith 2009, p. 54)

There is clearly something relevant in this distinction: when most fitness differences are due to sheer spatio-temporal accidents (Godfrey-Smith gives the classical example of the lightning bolt), evolution by natural selection is greatly hampered ${ }^{10}$.

Godfrey-Smith mentions the dependence of fitness differences on intrinsic characters $(S)$ as an essential reason why our somatic cells are not paradigmatic Darwinian populations:

"But another factor is the suppression of $S$. Much of what determines whether a cell divides or not is how it is located in relation to other things, which inundate it with signals, control its nutrients, and interfere with it if it behaves abnormally. Cell fitness is not very closely tied to intrinsic

10 As Godfrey-Smith acknowledges, the notion of intrinsicality is philosophically very problematic. I believe it is not the best way to make sense of this dimension. Wholly external fitness differences can lead to evolution by natural selection (see for instance Bouchard 2008), and a better way to understand the dimension would be in terms of the stability of the fitness differences over lineages. However, as the full elaboration and defence of this account is beyond the scope of this paper, I will here keep to Godfrey-Smith's characterization of the dimension. I therefore assume that in most cases, the dependence of fitness differences on intrinsic characters is a reliable proxy for the stability of the differences. 
Our somatic cells are de-Darwinized populations, at least in part because of a very low $S$. In contrast, he continues, cancer cells are re-Darwinized by increasing $S$. Godfrey-Smith's story is in line with the hallmarks of cancer previously mentioned, among which one can find the self-sufficiency in growth signals and insensitivity to inhibition signals. These features make the fitness of individual cancer cells (within the organism's life) less dependent on outside signals and more dependent on intrinsic features.

This account, while not erroneous, is incomplete. The importance of the tumour microenvironment is currently being emphasized in different fields of cancer biology. For example, it has been shown that cancer cells are "normalized" to their context, in other words they are transformed by their environment to conform to the tissue in which they are present (Rubin 2006). A particularly relevant case where the micro-environment plays a major role in the fate of cells is stem cell niches. In most tissues, stem cells are located in a particular micro-environment (e.g. the bone marrow for hematopoeitic stem cells) that protects them and maintains them in an undifferentiated state. Recently, the role of the niche in the maintenance of stem cells has been emphasized by a number of studies showing, among other things, the capacity of the niche to de-differentiate somatic cells back to stem cells (Voog and Jones 2010; see also Kai and Spradling 2004). Assuming that the tumorigenic mutation occurs in a stem cell, the niche could protect it, ensure its self-renewal (notably by keeping it undifferentiated) and stimulate its proliferation (especially in response to injury). But it also means that the niche could transform a differentiated tumour cell into a CSC just because it happened to be there. Being a CSC might not be wholly intrinsic to the cell, but might be extrinsic to a large extent. The most relevant fitness differences between cells, then, could be due to spatio-temporal accidents.

In fact, the extent to which the fate of cancer cells depends on their environment is so great that it seems at odds with the received view on carcinogenesis. As mentioned earlier, the traditional model of carcinogenesis states that cancer is caused by the accumulation of semi-specific mutations. Some authors, however, suggest that these mutations are merely permissive rather than causative (Huang 2011; see also Heng et al. 2010). While this view is not widespread, some important phenomena seem to support it. First, it has been demonstrated that transformation and hyperproliferation can be induced (with complete penetrance) in epithelial cells through genetic intervention on adjacent cells of the micro-environment (Bhowmick et al. 2004; see Bhowmick and Neilson 2004 for a review). Likewise, it seems that the tumorigenic phenotype can be reverted much more easily than the received view would suggest: some cancer cells, when injected in an embryo, lose their cancerous phenotype (Bussard et al. 2010; Gardner 1975). The same "reprogramming" can be done with oocyte extracts (Allegrucci et al. 2011) or by transplantation of the nucleus of cancer cells into oocytes (Hochedlinger et al. 2004). Furthermore, it has been reported that in some cases the cancer cells injected in embryos were compatible with the normal embryonic development (Mintz and Illmensee 1975) ${ }^{11}$. Such major phenotypic transformation is not limited to drastic laboratory manipulations, but has also been shown to occur within a tumour: for instance, changes in the stroma strongly modulate tumour growth (Bissell and Radisky 2001; Boudreau et al. 1995). Obviously, since these changes restrain proliferation, they decrease the fitness of the cells. If cues from the micro-environment determine much of the fitness of cells, then the situation is not so different from normal somatic cells.

Godfrey-Smith is certainly right that cancer cells are less determined by their environment than

11 Since some years later Karl Illmensee fraudulently reported the cloning of mice (see Kolata 1998), one should be careful in interpreting the results of Mintz and Illmensee's (1975). Hochedlinger et al. (2004) were able to obtain viable chimeric mice by transplanting the nucleus of melanoma cells into oocytes, but these mice showed a very high cancer susceptibility. 
normal cells, but to picture them as self-determined would be a mistake. $S$ is significantly higher in cancer cells than in normal somatic cells, but it is far from being maximal.

\section{Taking stock}

Let us now summarize our evaluation of cancer cells. Cancer cells have sufficient variation $(V)$ for evolutionary change, and although their relatively low fidelity of heredity $(H)$ might threaten the possibility of complex adaptation, it is generally in the range compatible with minimal Darwinian processes. However, cancer cells display a level of integration $(I)$ reminiscent of organs, and share reproductive fate to an important extent. Much of the fitness differences between cells does not depend on their intrinsic features $(S)$, which strongly suggests that these differences cannot be the basis for paradigmatic Darwinian processes. Perhaps the most crucial dimension, the degree of reproductive specialization $(G)$ will vary from case to case, depending on the extent to which a given cancer follows a CSC model. When it does, long-term proliferation will be restricted to a tiny sub-population of the tumour $(G)$, rendering most cancer cells unable to accumulate evolutionary changes. A very schematic assessment of the dimensions is represented in Table 1.

\begin{tabular}{|c|c|c|c|c|c|c|}
\hline Population & $\mathbf{F}$ & $\mathbf{V}$ & $\mathbf{C}$ & $\mathbf{I}$ & G & $\mathbf{S}$ \\
\hline Paradigmatic Darwinian population & +++ & + & +++ & - & -- & +++ \\
\hline Healthy somatic cells & +++ & - & ++ & ++ & ++ & -- \\
\hline Cancer cells: & ++ & + & + & + & $+/-$ & - \\
\hline $\begin{array}{l}\text { - CSC-model } \\
\text { (e.g. myeloid leukaemia) }\end{array}$ & ++ & + & + & + & ++ & - \\
\hline $\begin{array}{l}\text { - Non-CSC } \\
\text { (e.g. melanoma) }\end{array}$ & + & ++ & + & + & - & + \\
\hline
\end{tabular}

Table 1: Schematic position of key populations in Godfrey-Smith's spatial framework. A “+” represents a higher value, and "-" a lower value.

It should be uncontroversial by now that cancer cells are in general at least a minimal Darwinian population: they have the features required to undergo natural selection. Indeed, as mentioned earlier, tumour archaeology is best explained by natural selection. However, the observations made in the previous sections have shown that the typical picture of the cancer cell as a renegade cell, freed from the de-Darwinizing features of our somatic cells, is over-simplified. The exact position of cancer cells in Godfrey-Smith's space will vary according to the type of cancer, with the degree to which it follows a CSC model being of particular relevance. In general, although cancer cells are more Darwinian than healthy somatic cells, the difference between them should not be exaggerated. Especially, the tissue organization of cancer cells and the dependence of their fitness differences on their microenvironment should not be underestimated. Cancer cells are not paradigmatic Darwinian populations (although some forms of cancer are closer to it than others). According to Godfrey-Smith's framework, therefore, these processes are unlikely to lead to complex adaptation. In the second part of this paper, I investigate whether this is the case by discussing the best candidates for adaptation in cancer cells, and attempt to evaluate the explanatory relevance of natural selection in this context. 


\section{How important is natural selection to understand cancer cells?}

Cancer cells are at least minimal Darwinian populations, but not paradigmatic ones. This suggests that although we should expect Darwinian processes to occur (as they do), we should not expect too much out of those processes. According to Godfrey-Smith, paradigmatic Darwinian populations

"are the ones that have great scientific importance. These are the evolving populations in which significant novelty can emerge, the ones that give rise to complex and adapted structures." (Godfrey-Smith 2009, p. 41)

Complexity is notoriously difficult to define, but it becomes more tractable if we think of adaptation as a process rather than as an outcome, and consider complex adaptations are the product of cumulative evolution. As Godfrey-Smith suggests, minimal Darwinian populations are unlikely to give rise to complex adaptations because they offer "little possibility for "cumulative" change, for evolutionary processes involving the successive addition of slight modifications to an existing structure." (GodfreySmith 2009, p.45).

The idea that such an accumulation distinguishes evolution by natural selection (i.e paradigmatic Darwinian processes) from minimal Darwinian processes is present at least implicitly in many strands of philosophy of biology. In optimality models, local hill-climbing is paradigmatic of adaptations, and "climbing" implies the accumulation of "steps". More explicitly, Hull et al. (2001), who proposed an account of evolution based on processes rather than on conditions or features of populations, considered that without a strong iterative component, a process should not be called evolution by natural selection. Sterelny $(2007$; 2006) makes a similar point in his discussion of evolvability, stating that " $[\mathrm{t}]$ he crucial point to remember is that significant evolutionary change typically depends on the accumulation of smaller changes" (Sterelny 2007, p.13). To say that natural selection explains complex adaptations through the accumulation of small changes has nothing to do with gradualism: there is no need for this process to be very slow or even regular, nor for the different steps to be similar in magnitude. Simply, it has to be cumulative in order to produce complex adaptations. As I will show in the next section, the notion of accumulation can be used to map the relative importance of natural selection in the explanation of the resulting adaptation.

\section{The explanatory role of natural selection: a paradigmatic example}

The explanatory role of natural selection extends much beyond the simple spread of beneficial variations. As Ridley repeatedly emphasized in his famous textbook on evolution, "natural selection is the only explanation for adaptation" (Ridley 2007, p. 256, 259). It would have been more correct, however, to say that natural selection is the only explanation for complex adaptations. To illustrate this, consider first a toy example, which I will supplement in a moment with a real case. Both giraffes and humans with the condition of acromegaly (let us call them "giants") are unusually tall. But the actual difference-makers (Waters 2007) differ. If we compare the giants with humans that have a "normal" height, we will notice that the only consistent difference is a mutation in the NSD1 gene. If we compared, however, the giraffes with their short ancestors, we would most probably notice that their difference in height is due to the accumulation of a broader array of changes. The point is that without natural selection, a mutation in the NSD1 gene is very unlikely, but the accumulation of all the changes making the difference for the giraffe's height is incommensurately less likely. Independently of whether or not acromegaly is fitter than the normal condition, we could expect it to arise once in a while by chance. But without natural selection, we would never expect giraffes to arise from short ancestors by 
chance. This is, I believe, the intuition behind Ridely's statement: while mundane adaptations could be accidental, natural selection is the only explanation for complex adaptations.

Let us now examine the relevance of this point more thoroughly in the context of a real case. The threespine stickleback (Gasterosteus aculeatus) is often presented as a paradigmatic example of evolution by natural selection. Initially a salt-water fish found throughout the world, it started colonizing different fresh-water areas after the retreat of the glaciers. Different populations adapted to their new habitats in strikingly consistent manners, offering a recent example of convergent evolution. One such adaptation is the loss of lateral plates (or reduction of body armour). Although it is not entirely clear why (Kingsely and Peichel 2007), it is beneficial to have less plates in the fresh-water environment. Salt-water populations are almost completely plated (up to 36 plates each side), while fresh-water populations have very few plates, and different crosses produce intermediate amounts of plates (Kingsely and Peichel 2007). Using quantitative trait loci (QTL) analysis, a method similar to a simple analysis of variance, these variations were mapped to four different genetic loci: a major one accounting for up to $75 \%$ of the variation in plate number, along with three minor loci (Colosimo et al. 2004). These different loci are not straightforwardly additive, but all have an independent effect. The major locus was later identified to be in the Ectodysplasin gene - Eda (Colosimo et al. 2005). ${ }^{12}$

The chances for an organism to randomly acquire the variation in Eda are low, but the chances to randomly acquire the variations at the four loci, on both alleles, are orders of magnitude lower. Natural selection, however, makes it much more likely. Since, as was observed, each locus has at least a small effect in reducing the number of plates, it can be assumed that any of these variations, if it appears in a fresh-water environment, will tend to spread through the population. If each mutation arises in some individual and then spreads to the population, it becomes much more likely for the whole combination to appear in a single individual. Natural selection, therefore, is essential to the explanation as it alone can put the explanandum within a reasonable range of likelihood.

Imagine, on the other hand, a single-locus scenario in which all observed variation in plate numbers would be accounted for by the variation in Eda only. Natural selection occurs and has an explanatory role in both scenarios, but its explanatory power is much greater in the first case. The main difference between the two scenarios is that in the multi-loci case, the trait is a coherent accumulation of changes through multiple iterations of natural selection ${ }^{13}$. It is for the explanation of these complex adaptations that paradigmatic Darwinian processes are essential. For more mundane adaptations, which are not the results of cumulative evolution, alternative explanations might be more relevant. The important question, therefore, is whether adaptations in cancer cells are accumulated features or not.

\section{Four candidate adaptations in explanations in cancer}

It is often held that "the consistent phenotypical changes that emerge during carcinogenesis must always represent successful adaptations", and that their "genotypes and phenotypes can be understood according to their roles as adaptive strategies" (Gatenby and Gillies 2008, p. 56, emphasis

12 For the sake of simplicity, in what follows I make the reasonable assumption that each locus was, throughout the evolution of fresh-water sticklebacks, modified respectively through one mutational event. Should it turn out to be different, it would only make the case stronger.

13 By cycle or iteration, I do not mean a single generation or reproductive event: rather, it can encompass rounds of natural selection up to the fixation of a variant. 
added). There are indeed serious candidates for adaptations: cancer cells avoid immune surveillance, and tumour relapses suggest that they adapt to therapy. Some would even claim that the very phenomenon of carcinogenesis requires an adaptive explanation (see Hanahan and Weinberg 2000). In this part of the paper, I therefore turn to the most important of these, and assess the importance of natural selection in their explanation. In order to do this, I ask in each case whether the adaptation is an accumulation of evolutionary change, and explore alternative explanations.

\section{Evasion of senescence and apoptosis}

Upon stress, most notably genetic damage, normal somatic cells undergo either senescence (stop replicating) or apoptosis (programmed cell death). As I mentioned earlier, cancer cells have considerable genetic damage, and yet continue proliferating. Somehow, therefore, they must avoid this normal control mechanism, and this is among the most important hallmarks a cell must acquire to be cancerous. Very often, this is done by mutating the gene TP53: by far the most famous tumour suppressor, it is mutated in over half of all tumours. A cell that has lost p53 activity will enjoy a tremendous advantage over its counterparts. Natural selection will occur very straightforwardly: cells without p53 activity will proliferate while the others, ceteris paribus, will stall or die. But this is not the result of cumulative evolution. The structure of the transduction pathways regulating apoptosis makes it sufficient to remove this specific node (p53) from the network to effectively disable the control mechanism. The point is made even stronger by the fact that p53 mutations are often dominant negatives (Srivastava et al. 1993; De Vries et al. 2002), which means that a mutation in a single copy of the gene is sufficient to prevent the functional copy from doing its work (the mutated protein acts as a decoy). In other words, it is not even necessary to mutate both alleles, but a single change is sufficient. Knowing this, and looking at the wiring diagram of regulation of apoptosis, p53 appears as an obvious weak spot ${ }^{14}$. Among all alterations that would have been beneficial for the cell, this was a particularly easy one to get. Therefore, not only is there no accumulation, but there is also an architectural explanation: the original wiring of the cell leads us to expect, to some extent, the loss of this control mechanism.

Contrast this with an alternative scenario in which the molecular network would have a high degree of redundancy, for instance by having several copies of the gene encoding for p53 (and without dominant negative). In such a context, the original wiring of the cell would not lead us to expect the loss of the control mechanism. Should it happen, natural selection would have a lot more to explain indeed it would have a great deal more to accomplish. In order to lose all copies or functional equivalents, cells would have to accumulate a series of changes through many cycles of natural selection. If most of these changes, taken individually, make the control mechanisms weaker, each of them can be expected to spread. Their accumulation would therefore require an adaptive explanation. In the actual scenario, however, cancer cells' evasion of apoptosis and senescence is not a complex adaptation: it is not the result of cumulative evolution, and there are alternative, architectural reasons to expect it to arise. Of course, p53 mutation is not always the cause of this cancerous feature, and other cases might feature a higher degree of accumulation, but p53 is by far the most common culprit.

\section{Immunoediting and immune evasion}

It is a common idea that cancer cells escape immune recognition, and since our immune system is adaptive, this seems to require that cancer cells continuously evolve escape mechanisms. This

14 Of course, it would have been historically unthinkable to discover the importance of p53 mutations in this way, but this is altogether another question. 
conclusion rests on the assumption that if tumours did not evolve such mechanisms, they would be held in check by the immune system. This is not so obvious (Khong 2002); after all, cancer cells are our cells, not foreign pathogens, and recognizing them from healthy cells is no easy task. As a matter of fact, the immune system very often helps tumour progression, for instance by clearing dying cells (see Gregory and Pound 2011). Nevertheless, there is strong evidence that cancer cells escape immune recognition, and the most famous way through which this is done is the loss of the Human Leukocyte Antigen (HLA) system. These proteins are at the surface of cells and present antigens to patrolling Tcells. Without them, the cells do not present anything and therefore avoid recognition by an important portion of the immune system. Assuming that the immune system could normally recognize the cancer in this way, the loss of the HLA would be highly adaptive, and one would have to concede that through natural selection (immune selection), such an "adaptation" would spread through the tumour. But could we really expect the immune system to overcome or sidestep this problem? Will we really see an immune answer, leading into a cyclic arms-race? Apparently, the short is no:

"If cycles of immune pressure and tumor escape were operative during tumor development, one might expect to observe (either by examination or by imaging modalities) progressive tumor growth that was interspersed with one or more periods of contraction. Ongoing immunological 'shaping' or 'sculpting' of tumors might be expected to result in the destruction of sensitive cells followed by proliferation of immunoresistant cells that would, in turn, form the bulk of a new tumor emerging from the bed of the old tumor. However, solid tumors generally do not have growth curves with evidence of significant drops or depressions: tumors simply grow, and then grow larger.

[...] Although immune cells can be observed in or around tumors, spontaneous local inflammation in an uninfected tumor is generally not seen clinically or histologically.” (Khong 2002, p. 3)

Although we do observe selected changes, such as the loss of the HLA molecules, they are rather oneshot changes: there is little evidence of an iterative process of natural selection, much less of meaningful accumulation of evolutionary change.

\section{Resistance to therapy}

The best candidate adaptation in cancer cells is certainly resistance to therapy, analogous to bacterial development of resistance against antibacterial agents (Goldie and Coldman 1984). There are three major ways in which cancer cells acquire resistance to drugs; each of them, I argue, requires little or no accumulation of evolutionary change.

The first is quiescence: since most chemotherapeutic agents (e.g. anthracyclines or mustard gas) destroy replicating cells, a simple strategy is to avoid replicating too often. The second way is more complex, and consists in preventing drug uptake - in other words preventing the drug to get in the cell, or evacuating it. This is most notably done through the activation of the ATP-Binding Cassette (ABC) transporters. It is important to note that these first two strategies are both features of at least some of our (non-cancerous) cells under normal physiological conditions. Normal stem cells, for instance, prevent DNA damage in part by remaining relatively quiescent, and chemical cleansing (mainly through the ABC efflux pumps) is a characteristic feature of mesenchymal cells (Singh and Settleman 2010). In fact, as Huang notes, "[t]he rapid development of drug resistance can [...] be seen as a manifestation of an embryonic program since immature cells express an efficient detoxification program." (Huang 2011, p. 194). The same explanation is given within the CSC model described earlier, according to which some cancer cells, akin to normal stem cells, are quiescent and protected by their niche. The CSC model (as well as Huang's own model of cancer attractor) fully accounts for this kind of resistance and for the associated tumour relapse. Because the mechanism was already there, 
waiting to be activated, its appearance in cancer cells is much less surprising.

A third way to resist drugs applies mostly to targeted therapies, and implies reducing the expression of a target or intermediate. Hormone therapy, for instance Tamoxifen, targets an estrogen receptor; likewise, immunotherapy targets antigens, and all other targeted drugs either target a specific element in a pathway, typically to disrupt it, or are toxic only in the presence of a specific element. In all cases we have the same response: if there is any way for the cell to be alive without expressing the target, it can simply lose it to acquire resistance. To give only one of the many available examples, it was recently shown that melanoma resistance to vemurafenib is due to a loss of exons necessary for the drug's effect (Poulikakos et al. 2011). A cell that has lost the target of a drug with which the patient undergoes therapy, provided that it is still viable, will be selected for and increase in relative concentration. Again, this is clearly natural selection, but it is not a complex adaptation: they are rather one-shot changes that require no accumulation. In fact, very sensitive methods have detected that the mutations providing the resistance to a therapy are present before the beginning of the treatment (e.g Roche-Lestienne et al. 2002 for Imatinib) ${ }^{15}$.

In all three cases, cancer cells' resistance to a therapy is not the result of cumulative evolution. Instead, the features were somehow always there, already wired in the architecture of the normal cell. But what about resistance to multiple therapies? The question prompts a deeper concern: what if we adopt another level of granularity with respect to traits?

\section{The problem of granularity: multi-resistance and cancer initiation}

Let us assume that I am right in that neither evasion of the adaptive immune system nor the loss of DNA-damage checkpoint are the result of cumulative evolution. The conclusion might hold only because we think of immune recognition and senescence/apoptosis as two independent selection pressures. If we adopt a more general perspective and consider both selection barriers as one, for instance "cell control", then these two changes are accumulated in developing a slightly more complex adaptation to circumvent "cell control". There is no way to "cut traits at the joints", and the distinction between accumulating different adaptations and accumulating different changes toward the same adaptation is not ontological. We must therefore accept that the amount of accumulation will vary according to the level of granularity. Hence I would grant multi-resistance, provided that the resistance to each therapy does not stem from a common alteration, is the result of cumulative evolution and therefore that natural selection is essential in explaining it. However, two points must be noted with regard to this case.

First, one has to be clear about what exactly is being explained: natural selection explains the accumulation of different resistances, but it explains very little of the resistance to a given therapy. Nor does it suggest whether resistance to a given therapy is likely to develop or not.

Second, the kind of cumulative evolution responsible for multi-resistance has an important difference with paradigmatic cases of cumulative evolution like the stickleback. Multi-resistance is the result of exposure to a series of therapies, and presupposes that a resistance mechanism evolves for each therapy. But unlike the four loci in the example of the stickleback, these mechanisms are not interchangeable: if resistance to the fourth therapy had arisen during treatment with the first therapy, it would not have been selected for. This means that although natural selection makes the whole combination more likely by fixing the first ones in the population, the relative increase in likelihood is considerably lower than in the case of the stickleback, where any of the four loci has an autonomous effect of the reduction of plates. In other words, natural selection does play an important explanatory

15 A recent report also suggests that the stroma commonly confers an "innate resistance" - see Straussman et al. (2012). 
role, but not as important as it could be - and it leaves a lot to be explained.

Taking yet another level of granularity, one could consider the cancerous phenotype as a whole to be an adaptation. Indeed, it has been argued that cancer initiation cannot be explained without natural selection. The multiple-hit theory of carcinogenesis states that cells must acquire a series of (semi-)specific mutations to becomes cancerous, but many authors have noted that this is difficult to reconcile with the known lifespan and mutation rates of most somatic cells (for instance Smalley and Herlyn 2009, p. 1245). Given the frequency of cancer, it seems too unlikely to gather the combination of mutations in the short lifespan of a single somatic cell. Hence natural selection was proposed to account for this: if the first hit is already advantageous for the cell, it will be spread through the population, making the combination of hits more likely by changing the background conditions on which the next hit will arise ${ }^{16}$. A related - and still very much debated - hypothesis is that of the "mutator phenotype" (Beckman and Loeb 2006; Loeb 1991), according to which one of the first hits increases genetic instability and thereby makes subsequent hits more likely. Once more, however, the CSC model provides an alternative explanation which is equally convincing: if cancer either arises in a stem cell or revert the cell to a pre-wired stem-cell state, the cell has the necessary lifespan. Moreover, many of the hallmarks of cancer are physiological features of stem cells ${ }^{17}$. Nevertheless, not all cancers follow a CSC model, and depending on the case at hand it is likely that carcinogenesis as a whole is a cumulative evolution. Once more, it is important to be clear about what exactly is being explained: it is neither the immortality of the cancer cell, nor its evasion of immune surveillance, nor any of its hallmarks. It is only that all the beneficial features, whatever they are, have accumulated.

\section{Taking stock}

For cancers that do not follow a CSC model, the process of carcinogenesis as a whole is the best candidate for cumulative evolution. This is consistent with the observation that these cancers are more Darwinian than cancers following a CSC model. Natural selection can potentially explain why cancer is likely despite the low likelihood of randomly accumulating the necessary changes, but it does little more.

The other candidate adaptations reviewed here are definitely not complex adaptations. They have an important thing in common: they all consist in the loss of something (generally, of a regulatory mechanism). As Huang puts it, in the context of cancer mutations "are not constructive but destructive in their action" (Huang 2011, p. 193). Indeed, it seems that cancer cells are limited to either activating dormant features or losing evolved ones, and do not develop genuinely new traits - which is, according to Gofrey-Smith, the "mark of a significant Darwinian process." (Godfrey-Smith 2009, p. 43). Of course, logically a loss is equivalent to a gain of the opposite trait. The point is more about the complexity of the adaptations: the losses occurring in cancer cells all seem one-shot changes rather than perfectible, accumulated features. This is consistent with the observation, in the first part of the paper, that cancer cells are not a paradigmatic Darwinian population. Moreover, it suggests that alternative explanation might be more relevant. Invoking selection removes very little of the surprise at seeing cancer cells evacuate drugs so efficiently. By contrast, showing that mesenchymal cells already possess the tools for evacuating chemicals removes a great deal of surprise at seeing this behaviour in cancer cells. Far from an isolated case, this seems to be the norm in cancer: to give another example, it has recently been demonstrated that the invasive behaviour characteristic of melanoma cells is a

16 While there are cases in which all changes appeared together in a single event of massive mutation (Stephens et al. 2011), they are arguably very rare.

17 Likewise, Huang's (2011) model of unused attractor states offers an equally satisfying explanation. 
reactivation of the embryonic migration programme in the neural crest (Bailey et al. 2012). Carcinogenesis itself is considered to be analog to wound healing (Bissell and Radisky 2001). Certainly, the re-activation has to be due to something, and natural selection is necessary to explain the spread of the variation, but in relation to other components of the explanation, its importance has been greatly over-rated.

\section{Conclusion}

In the first part of this paper, I have argued that cancer cells are not paradigmatic Darwinian populations according to Godfrey-Smith's framework. Some types of cancer - most significantly those that do not follow a CSC model - are more Darwinian than others, but in general cancer cells are much farther away from paradigmatic Darwinian populations, and much closer to normal somatic cells, than is generally assumed. Consistent with this conclusion, most adaptations (and arguably the most important adaptations) in cancer cells are not complex adaptations, in other words they are not the result of cumulative evolution, but rather like one-step changes. For this reason, natural selection is not the essential component in their explanations. Instead, it is the pre-existing wiring of the cell which best accounts for these features. This is not to deny that the changes are selected for, but simply to say that the most enlightening explanatory material is already inside the cell, akin to architectural constraints. The healthy cells - their structure, possible states, pathways, and weak spots - already contain the resources to be drawn upon and developed by cancer cells. It dictates their evolution to a large extent.

The exercise undertaken here has shown the fruitfulness of Godfrey-Smith's framework in two ways. First, the framework was successful in guiding the search, organization and analysis of the empirical data relevant to the somatic evolution of cancer cells. More importantly, the predictions of Godfrey-Smith's framework have been corroborated. Since cancer cells are minimal Darwinian populations but not paradigmatic Darwinian populations, they were expected to undergo natural selection but not to develop complex adaptations. In the second part of this paper, I argued that this is indeed the case: while there is strong evidence for the action of natural selection, there does not seem to be anything near complex adaptations, with the possible exception of carcinogenesis taken as a whole in cancers that do not follow a CSC model. Interestingly, this last exception is related to types of cancer which are, following Godfrey-Smith's framework, more Darwinian than those following a CSC model. As far as this case is concerned, the framework is consistent with the facts.

I started the paper quoting scientists who claim that "the fundamental problems of neoplastic progression and cancer therapy are also problems of evolutionary biology" (Merlo et al. 2006, p. 933). It has been argued that the mechanisms underlying cancer, because they are the result of Darwinian processes, are so diverse that it would be impossible to catalogue them all (Heng et al. 2010, p. 1080), and therefore that traditional molecular oncology is doomed to fail. Many authors claim, for instance, that the emergence of resistance is predictable on the ground of natural selection (see Gillies et al. 2012, p. 490). However, as I have tried to show throughout the paper, cancer cells cannot (see the first part of the paper) and do not (see the second part) evolve complex adaptations: they are limited to those adaptations that are made immediately possible by their original molecular architecture. This architecture, therefore, and the possibilities it offers, are better predictors of the emergence of resistance than the mere presence of minimal Darwinian processes. Evolution by natural selection plays a role in neoplastic progression and in the acquisition of resistance, but "the fundamental problem of cancer", if there is such a thing, is more a problem of cells whose inner workings have vulnerabilities. It is the 
problem of cells which have an inherent potential to become cancerous and resistant to some therapies.

Nevertheless, the dynamic heterogeneity of cancer poses serious challenges to its treatment, and (independently of evolutionary accounts of cancer) the medical community is increasingly turning to prevention (see for instance the last American "report to the Nation", Eheman et al. 2012). However, one should not confuse the public health question of whether it is more effective to invest in prevention or treatment, with the biomedical question of how best to understand cancer and treat its victims. In answering the biomedical question, it is important to recognize some of the spectacular results of molecular oncology, and to persevere in what is arguably the best way of studying cancer biology.

It is telling that a very recent and extremely interesting study on tumour archaeology, even though it starts by noting that "[c]ancer evolves dynamically as clonal expansions supersede one another driven by shifting selective pressures" (Nik-Zainal et al. 2012b, p. 994), contains virtually no mention of natural selection. Discussion of selection or fitness occurs exclusively in the very theoretical introduction, and the notions are mentioned nowhere else in the paper, let alone in the discussion of the data. As this example suggests, natural selection seems useful to make sense, abstractly, of the big picture, but we should not expect too much from it. Although an evolutionary approach should be integrated to our general understanding of cancer (as is currently being done), I believe it should not reorient cancer biology in a major way.

\section{Acknowledgements}

I am particularly indebted to Mark A. Bedau and Fridolin Groß, who offered very precious assistance in this project, as well as to an anonymous reviewer who was particularly helpful. In addition, I would like to thank all those who took the time to read and comment any of the countless drafts of this paper: Giuseppe Testa, Michel Morange, Pierre-Olivier Methot, Giovanni Boniolo, Marcel Weber, Lorenzo Del Savio, Annette Kappeler, Marco Annoni, Cecilia Nardini and Matteo Mamelli. Finally, I owe to the European School of Molecular Medicine (SEMM), the IFOM-IEO Campus and the FOLSATEC program the chance to have delved deeper into this science.

\section{Bibliography}

Adams JM, Strasser A (2008) Is Tumor Growth Sustained by Rare Cancer Stem Cells or Dominant Clones? Cancer Research 68:4018-4021. doi:10.1158/0008-5472.CAN-07-6334

Allegrucci C, Rushton MD, Dixon JE, et al. (2011) Epigenetic Reprogramming of Breast Cancer Cells with Oocyte Extracts. Molecular Cancer 10:7. doi:10.1186/1476-4598-10-7

Anderson K, Lutz C, van Delft FW, et al. (2011) Genetic Variegation of Clonal Architecture and Propagating Cells in Leukaemia. Nature 469:356-61. doi:10.1038/nature09650.

Armitage P, Doll R (1957) A Two-Stage Theory of Carcinogenesis in Relation to the Age Distribution of Human Cancer. British Journal of Cancer 11:161-169.

Attolini CS-O, Michor F (2009) Evolutionary Theory of Cancer. Annals of the New York Academy of Sciences 1168:23-51. doi:10.1111/j.1749-6632.2009.04880.x 
Bailar JC, Smith EM (1986) Progress against Cancer? New England Journal of Medicine 314:1226-1232. doi:10.1056/NEJM198605083141905

Bailey CM, Morrison J, Kulesa PM (2012) Melanoma Revives an Embryonic Migration Program to Promote Plasticity and Invasion. Pigment Cell \& Melanoma Research. doi:10.1111/j.1755-148X.2012.01025.x

Barker N, Ridgway RA, van Es JH, et al. (2009) Crypt Stem Cells as the Cells-of-Origin of Intestinal Cancer. Nature 457:608-6. doi:10.1038/nature07602

Beckman RA, Loeb LA (2006) Efficiency of Carcinogenesis with and without a Mutator Mutation. Proceedings of the National Academy of Sciences 103:14140-5. doi:10.1073/pnas.0606271103

Belov K (2012) Contagious Cancer: Lessons from the Devil and the Dog. BioEssays 34:285-292. doi:10.1002/bies.201100161

Bertolaso M (2011) Hierarchies and Causal Relationships in Interpretative Models of the Neoplastic Process. History and philosophy of the life sciences 33:515-5138.

Bhowmick N, Chytil A, Plieth D, et al. (2004) TGF-beta Signaling in Fibroblasts Modulates the Oncogenic Potential of Adjacent Epithelia. Science 303:848-51. doi:10.1126/science.1090922

Bhowmick N, Neilson E (2004) Stromal Fibroblasts in Cancer Initiation and Progression. Nature 432:332-337

Bissell MJ, Radisky D (2001) Putting Tumours in Context. Nature reviews. Cancer 1:46-54. doi:10.1038/35094059

Bouchard F (2008) Causal Processes, Fitness, and the Differential Persistence of Lineages. Philosophy of Science 75(5):560-570. doi:10.1086/594507

Boudreau N, Sympson CJ, Werb Z, Bissell MJ (1995) Suppression of ICE and Apoptosis in Mammary Epithelial Cells by Extracellular Matrix. Science 267:891-893.

Buss LW (1987) The Evolution of Individuality. Princeton University Press 201.

Bussard KM, Boulanger C a, Booth BW, et al. (2010) Reprogramming Human Cancer Cells in the Mouse Mammary Gland. Cancer research 70:6336-43. doi:10.1158/0008-5472.CAN-10-0591

Cairns J (1975) Mutation Selection and the Natural History of Cancer. Nature 255:197-200.

Colosimo PF, Hosemann KE, Balabhadra S, et al. (2005) Widespread Parallel Evolution in Sticklebacks by Repeated Fixation of Ectodysplasin Alleles. Science 307:1928-33. doi:10.1126/science.1107239

Colosimo PF, Peichel CL, Nereng K, et al. (2004) The Genetic Architecture of Parallel Armor Plate Reduction in Threespine Sticklebacks. PLoS biology 2:E109. doi:10.1371/journal.pbio.0020109

Corada M, Zanetta L, Orsenigo F, et al. (2002) A Monoclonal Antibody to Vascular Endothelial-Cadherin Inhibits Tumor Angiogenesis without Side Effects on Endothelial Permeability. Blood 100:905-911.

Eheman C, Henley S, Ballard-Barbash R (2012) Annual Report to the Nation on the Status of Cancer, 1975 2008, Featuring Cancers Associated with Excess Weight and Lack of Sufficient Physical Activity. Cancer. doi:10.1002/cncr.27514

Frank NY, Schatton T, Frank MH (2010) The Therapeutic Promise of the Cancer Stem Cell Concept. Journal of Clinical Investigation 120:41-50.

Frank SA (2007) Dynamics of Cancer: Incidence, Inheritance, and Evolution. Princeton University Press

Frank SA, Iwasa Y, Nowak MA (2003) Patterns of Cell Division and the Risk of Cancer. Genetics 163:15271532. 
Gardner RL (1975) Fate of Teratocarcinoma Cells Injected into Early Mouse Embryos. Nature 258:70.

Gatenby RA, Gillies RJ (2008) A Microenvironmental Model of Carcinogenesis. Nature Reviews. Cancer 8:5661. doi:10.1038/nrc2255

Gerlinger M, Swanton C (2010) How Darwinian Models Inform Therapeutic Failure Initiated by Clonal Heterogeneity in Cancer Medicine. British journal of cancer 103:1139-43. doi:10.1038/sj.bjc.6605912

Gillies RJ, Verduzco D, Gatenby R a. (2012) Evolutionary Dynamics of Carcinogenesis and Why Targeted Therapy Does Not Work. Nature Reviews. Cancer 12:487-493. doi:10.1038/nrc3298

Godfrey-Smith P (2009) Darwinian Populations and Natural Selection. Oxford University Press

Goldie JH, Coldman AJ (1984) The Genetic Origin of Drug Resistance in Neoplasms: Implications for Systemic Therapy. Cancer Research 44:3643-3653.

Gould SJ, Lewontin RC (1979) The Spandrels of San Marco and the Panglossian Paradigm: A Critique of the Adaptationist Programme. Proceedings of the Royal Society of London Series B 205:581-598.

Gregory CD, Pound JD (2011) Cell Death in the Neighbourhood: Direct Microenvironmental Effects of Apoptosis in Normal and Neoplastic Tissues. The Journal of pathology 223:177-194. doi:10.1002/path.2792

Grompe M (2012) Tissue Stem Cells: New Tools and Functional Diversity. Cell Stem Cell 10:685-689. doi:10.1016/j.stem.2012.04.006

Hanahan D, Weinberg RA (2000) The Hallmarks of Cancer. Cell 100:57-70.

Hanahan D, Weinberg RA (2011) Hallmarks of Cancer: The Next Generation. Cell 144:646-674. doi:10.1016/j.cell.2011.02.013

Heng HHQ, Stevens JB, Bremer SW, et al. (2010) The Evolutionary Mechanism of Cancer. Journal of cellular biochemistry 109:1072-84. doi:10.1002/jcb.22497

Hochedlinger K, Blelloch R, Brennan C, et al. (2004) Reprogramming of a Melanoma Genome by Nuclear Transplantation. Genes \& development 18:1875-85. doi:10.1101/gad.1213504

Huang S (2011) On the Intrinsic Inevitability of Cancer: From Foetal to Fatal Attraction. Seminars in cancer biology 21:183-199. doi:10.1016/j.semcancer.2011.05.003

Hull DL, Langman RE, Glenn SS (2001) A General Account of Selection: Biology, Immunology, and Behavior. The Behavioral and brain sciences 24:511-28.

Kai T, Spradling A (2004) Differentiating Germ Cells Can Revert into Functional Stem Cells in Drosophila Melanogaster Ovaries. Nature 428:564-569.

Khong H (2002) Natural Selection of Tumor Variants in the Generation of "Tumor Escape" Phenotypes. Nature immunology 3:999-1005.

Kingsley DM, Peichel CL (2007) The Molecular Genetics of Evolutionary Change in Sticklebacks. Biology of the Threespine Stickleback. CRC Press, pp 4-81

Kolata G (1999) Clone: The Road to Dolly and the Path Ahead. Harper Perennial.

Leroi AM, Koufopanou V, Burt A (2003) Cancer Selection. Nature reviews. Cancer 3:226-31. doi:10.1038/nrc1016

Loeb LA (1991) Mutator Phenotype May Be Required for Multistage Carcinogenesis. Cancer Research 51:3075-3079. 
Maley CC, Galipeau PC, Finley JC, et al. (2006) Genetic Clonal Diversity Predicts Progression to Esophageal Adenocarcinoma. Nature genetics 38:468-73. doi:10.1038/ng1768.

Maenhaut C, Dumont JE, Roger PP, Van Staveren WCG (2010) Cancer Stem Cells: A Reality, a Myth, a Fuzzy Concept or a Misnomer? An Analysis. Carcinogenesis 31:149-158.

Merlo LMF, Pepper JW, Reid BJ, Maley CC (2006) Cancer as an Evolutionary and Ecological Process. Nature reviews Cancer 6:924-35. doi:10.1038/nrc2013

Miller SJ, Lavker RM, Sun T-T (2005) Interpreting Epithelial Cancer Biology in the Context of Stem Cells: Tumor Properties and Therapeutic Implications. Biochimica et biophysica acta 1756:25-52. doi:10.1016/j.bbcan.2005.07.003

Mintz B, Illmensee K (1975) Normal Genetically Mosaic Mice Produced from Malignant Teratocarcinoma Cells. Proceedings of the National Academy of Sciences of the United States of America 72:3585-3589.

Nagy JD (2004) Competition and Natural Selection in a Mathematical Model of Cancer. Bulletin of mathematical biology 66:663-87. doi:10.1016/j.bulm.2003.10.001

Nik-Zainal S, Alexandrov LB, Wedge DC, et al. (2012) Mutational Processes Molding the Genomes of 21 Breast Cancers. Cell 979-993. doi:10.1016/j.cell.2012.04.024

Nik-Zainal S, Van Loo P, Wedge DC, et al. (2012) The Life History of 21 Breast Cancers. Cell. doi:10.1016/j.cell.2012.04.023

Nowell PC (1976) The Clonal Evolution of Tumor Cell Populations. Science 194:23-28. doi:10.1126/science.959840

Pece S, Tosoni D, Confalonieri S, et al. (2010) Biological and Molecular Heterogeneity of Breast Cancers Correlates with their Cancer Stem Cell Content. Cell 140:62-73. doi:10.1016/j.cell.2009.12.007

Pepper JW, Findlay CS, Kassen R, et al. (2009) Cancer Research Meets Evolutionary Biology. Evolutionary Applications 2:62-70. doi:10.1111/j.1752-4571.2008.00063.x

Rabinovitch, P.S., Reid, B.J., Haggitt, R.C., Norwood, T.H. \& Rubin, C.E. (1989) Progression to Cancer in Barrett's Esophagus is Associated with Genomic Instability. Laboratory Investigation. 60:65-71.

Ridley M (2007) Evolution, 3rd Edition. Blackwell publishing

Roche-Lestienne C, Soenen-Cornu V, Grardel-Duflos N, et al. (2002) Several Types of Mutations of the Abl Gene Can Be Found in Chronic Myeloid Leukemia Patients Resistant to STI571, and they Can Pre-Exist to the Onset of Treatment. Blood 100:1014-1018.

Rubin H (2006) What Keeps Cells in Tissues Behaving Normally in the Face of Myriad Mutations? BioEssays 28:515-524. doi:10.1002/bies.20403

Sabatino M, Zhao Y, Voiculescu S, et al. (2008) Conservation of Genetic Alterations in Recurrent Melanoma Supports the Melanoma Stem Cell Hypothesis. Cancer Research 68:122-131.

Shibata D (2006) Clonal Diversity in Tumor Progression. Nature genetics 38:402-3. doi:10.1038/ng0406-402

Singer T, McConnell MJ, Marchetto MCN, et al. (2010) LINE-1 Retrotransposons: Mediators of Somatic Variation in Neuronal Genomes? Trends in Neurosciences 33:345-354.

Singh A, Settleman J (2010) EMT, Cancer Stem Cells and Drug Resistance: An Emerging Axis of Evil in the War on Cancer. Oncogene 29:4741-51. doi:10.1038/onc.2010.215

Singh SK, Clarke ID, Terasaki M, et al. (2003) Identification of a Cancer Stem Cell in Human Brain Tumors. Cancer Research 63:5821-8. doi:10.1038/nature03128 
Smalley KSM, Herlyn M (2009) Integrating Tumor-Initiating Cells into the Paradigm for Melanoma Targeted Therapy. International journal of cancer 124:1245-1250. doi:10.1002/ijc.24129

Soto AM, Sonnenschein C (2004) The Somatic Mutation Theory of Cancer: Growing Problems with the Paradigm? BioEssays 26:1097-107. doi:10.1002/bies.20087

Stephens PJ, Greenman CD, Fu B, et al. (2011) Massive Genomic Rearrangement Acquired in a Single Catastrophic Event During Cancer Development. Cell 144:27-40. doi:10.1016/j.cell.2010.11.055

Sterelny K (2007) What Is Evolvability? In: Matthen M, Stephens C (eds) The Elsevier Handbook of the Philosophy of Biology. Elsevier, pp 163-178

Sterelny K (2006) The Evolution and Evolvability of Culture. Mind \& language 21(2):137-165. doi: 10.1111/j.0268-1064.2006.00309.x

Stratton MR (2011) Exploring the Genomes of Cancer Cells: Progress and Promise. Science 331:1553-8. doi:10.1126/science. 1204040

Straussman R, Morikawa T, Shee K, et al. (2012) Tumour Micro-Environment Elicits Innate Resistance to RAF Inhibitors through HGF Secretion. Nature. doi:10.1038/nature11183

Visvader JE, Lindeman GJ (2012) Cancer Stem Cells: Current Status and Evolving Complexities. Cell Stem Cell 10:717-728. doi:10.1016/j.stem.2012.05.007

Vogelstein B, Kinzler KW (1993) The Multistep Nature of Cancer. Trends in Genetics 9:138-141.

Voog J, Jones DL (2010) Stem Cells and the Niche: A Dynamic Duo. Cell stem cell 6:103-115.

De Vries A, Flores ER, Miranda B, et al. (2002) Targeted Point Mutations of p53 Lead to Dominant-Negative Inhibition of Wild-Type p53 Function. Proceedings of the National Academy of Sciences 99:2948-2953.

Waters CK (2007) Causes that Make a Difference. The Journal of Philosophy 104(11):551-579.

Ye CJ, Stevens JB, Liu G, et al. (2009) Genome Based Cell Population Heterogeneity Promotes Tumorigenicity: the Evolutionary Mechanism of Cancer. Journal of cellular physiology 219:288-300. doi:10.1002/jcp.21663 